Cronfa - Swansea University Open Access Repository

This is an author produced version of a paper published in :

Trends in Ecology \& Evolution

Cronfa URL for this paper:

http://cronfa.swan.ac.uk/Record/cronfa31719

\title{
Paper:
}

Gallagher, A., Creel, S., Wilson, R. \& Cooke, S. (2017). Energy Landscapes and the Landscape of Fear. Trends in Ecology \& Evolution, 32(2), 88-96.

http://dx.doi.org/10.1016/j.tree.2016.10.010

This article is brought to you by Swansea University. Any person downloading material is agreeing to abide by the terms of the repository licence. Authors are personally responsible for adhering to publisher restrictions or conditions. When uploading content they are required to comply with their publisher agreement and the SHERPA RoMEO database to judge whether or not it is copyright safe to add this version of the paper to this repository. http://www.swansea.ac.uk/iss/researchsupport/cronfa-support/ 


\section{Energy landscapes and the landscape of fear}

2 Austin J. Gallagher ${ }^{1,2,3^{*}}$, Scott $\mathrm{Creel}^{4}$, Rory P. Wilson ${ }^{5}$, Steven J. Cooke ${ }^{1}$

$3 \quad{ }^{1}$ Fish Ecology and Conservation Physiology Laboratory, Department of Biology and Institute of

4 Environmental Science, Carleton University, Ottawa, ON K1S 5B6, Canada

$5 \quad{ }^{2}$ Rosenstiel School of Marine and Atmospheric Science, University of Miami, Miami, FL 33149,

6 USA

$7 \quad{ }^{3}$ Beneath the Waves, Inc., Miami, FL 33133, USA

$8{ }^{4}$ Department of Ecology, 310 Lewis Hall, Montana State University, Bozeman, MT 59717

$9 \quad{ }^{5}$ Biosciences, College of Science, Swansea University, Swansea SA2 8PP, UK

11 Abstract

12 Animals are not distributed randomly in space and time because their movement ecology is

13 influenced by a variety of factors. Energy landscapes and the landscape of fear have recently

14 emerged as largely independent paradigms, both re-shaping our perspectives and thinking about

15 the spatial ecology of animals across heterogeneous landscapes. We argue that these paradigms

16 are not distinct but rather complementary, collectively providing a better mechanistic basis for

17 understanding the spatial ecology and decision-making of wild animals. We discuss the

18 theoretical underpinnings of each paradigm and illuminate the complementary nature through

19 case studies, then integrate these concepts quantitatively by constructing models of movement

20 pathways modulated by energy and fear to elucidate the mechanisms underlying the spatial

21 ecology of wild animals.

23 Keywords: animal ecology; energy, fear, predators, movement 


\section{The mechanistic basis of animal movement}

The collective storing and interpretation of environmental information is a fundamental component of daily life at virtually all levels of organismal function and biological organization. For animals, this integration of information over time and space feeds into a complex decisionmaking process that drives behavioral changes critical to survival and fitness. The interest in this decision-making process, specifically as it relates to the ability to understand how animals move and are distributed through time and space, has fueled the study of animal ecology dating back to questions posed by Aristotle nearly 2,300 years ago [1]. selection explains why. Perhaps the most discussed driver for animal movement is foraging. Indeed, judicious harvesting of energy during foraging is what spawned the numerous publications on optimal foraging dating back to the 1970s where workers began by manipulating

41 and controlling resources in the laboratory [5]. The optimal foraging framework led to critical 42 conceptual advances in animal movement studies such as 'giving up time' and optimized 'central place foraging' which have since been applied to studies in the wild [3], changing the way the biological community thought about animal movement and prey selection [6]. acquisition, generally oversimplified environmentally dependent criteria, now considered

47 important for animal decision-making, such as energy loss during the very movement that is so 48 critical for resource acquisition [7], or exposure to predation risk. These omissions can limit the explanatory power of the approach because movement costs are highly variable (e.g., due to 50 physical properties of the environment) and typically involve among the greatest energy 
51 expenditures of animals [8][10], and it is now clear that the risk of predation can also structure

52 how animals use their landscapes [2,9]

The fact that important attributes of landscapes vary in both space and time has been the

54 central tenet of two separate and divergent research themes, both of which are today receiving

55 increasing attention in the research communities studying wild animal biology and ecology;

56 energy landscapes and the landscape of fear [2,10]. With respect to energy landscapes, recent

57 work has highlighted that the characteristics of the environment through which an animal moves,

58 irrespective of whether it is water, air or over ground, profoundly affect the power use of the

59 moving animal and therefore the costs of movement per meter travelled (the costs of transport)

$60[10,11]$. The landscape of fear, on the other hand, is grounded in the controlling effects predators

61 can have on prey, which trigger food vs. risk trade-offs which can change animal behavior

62 including movement $[12,13]$. Theory and methodological innovation (e.g., biotelemetry and

63 biologging) are at the core of both research themes. While they are both believed to have great

64 power in the ability to predict animal movement, each seems to be moving independently in

65 different directions although they should be integrated together to represent the real world. Here,

66 we propose to merge concepts central to energy landscapes with those relevant to the landscape

67 of fear to provide a framework that enhances our ability to understand how animals are

68 distributed in space and time. We briefly discuss the theoretical, biological, and ecological

69 underpinnings of both research themes, and illustrate the justification for their integration

70 through two real-world ecological examples. We then operationalize this idea by constructing

71 models of movement pathways modulated by energy and fear in hopes that our framework can

72 be used to calculate the amount of extra energy an animal is willing to spend to avoid predators

73 (i.e., the cost of predation risk and danger) [14] based on animal movement data. We hope that 
74 this framework will demonstrate potential for better understanding of why animals move and

75 how they are distributed in space and time.

\section{Energy landscapes}

The costs of movement depend on the environment through which an animal moves.

78 Although studies examining animal movement during migration have emphasized the

79 importance of barriers and flow streams (in air and water) in modulating movement [15,16], few

80 have demonstrated the role that these variable energy costs play in animal space use and

81 movement on a day to day basis [15,17]. Indeed, Dickenson et al. [18] note that determinations

82 of the costs of locomotion in a laboratory setting are unlikely to be applicable to the wild.

83 Unsurprisingly, therefore, where authors have examined how natural environments affect the

84 cost of locomotion, the variation in energy expenditure with environment type is impressive. For

85 example, we know that humans walking on 'soft sand' require 2.1-2.7 times more energy than on

86 solid rock $[19,20]$ and that people walking up slopes experience an increase in cost of transport

87 with slope angle such that a man walking up a $45^{\circ}$ slope expends 17 times more energy per

88 metre than walking on the flat [21]. Similarly, a bird flying into a wind at the speed of the wind

89 has infinite travel costs while if that same bird flies with the wind, it will have transport costs it

90 would have in still air.

So, given the ability to allude to the interaction between space, movement, energy

92 expenditure and behaviour in free-living animals, what might be expected for animals having to

93 operate in variable energy landscapes? Using an optimality approach, animals should respond to

94 energy landscapes to optimize energy expenditure over all time scales, for example on an hour to

95 hour or day to day basis, and their movement should reflect this. 
96 A generalized solution for the movement costs $(E E)$ between any two points can be represented

97 by:

98

99

100

101

102

103

104

105

107

108

109 113 of being predated.

$$
E E=\int P d t
$$

Where $\mathrm{P}=$ power. More properly though, power use would also be a function of the energy landscape and routine metabolic rate $(\mathrm{RMR}), \varepsilon$, so that

$$
P=R M R+f(\varepsilon)
$$

If, other things being equal, animals attempted to minimize travelling costs between two points, we would expect them to display a trajectory where the sum costs of all speeds and turns of the chosen trajectory across the different energy landscapes were minimized $\left(E E_{\min }\right)$ so that;

$$
E E_{\text {min }}=\operatorname{argmin}_{p}\left[\int_{t 0}^{t n} R M R+f(\varepsilon) d t\right]
$$

Here, the set of all possible paths through the landscape is represented by the set $\mathrm{P}$. The minimization cost function adds the resting metabolic cost (RMR) to the energy landscape cost ( $\varepsilon$ ) at all points along each path (from the beginning at $t_{0}$ to the end at $t_{n}$, the limits on the integration). The path with the lowest total value is the minimum cost path.

The formulation above provides a framework with which putative animal movement may be determined according to only one element though - energy. In addition, movement trajectories will depend on other things, notably the distribution of resources and the probability

\section{The landscape of fear}

In its initial representation, the 'landscape of fear' was based on predators eliciting, in their prey, a fear of being killed (the risk of predation) throughout the ecosystem [2]. These 'fear effects' can significantly alter the physiology, behavior, and life-history of prey species [22]. 
118 This "ecology of fear" is increasingly being recognized as crucial in understanding the role of

119 predators, the mechanics of predator-prey interactions, and even the ecosystem-wide

120 consequences of removing predators from natural systems [23]. It has been proposed that the

121 spatial and temporal manner in which wild animals utilize their landscapes is fear driven, and

122 that it permeates all areas of animal ecology [24]. This natural game of cat-and-mouse between

123 predators and their prey affects how both groups navigate their landscapes. This "landscape of

124 fear" interaction, which integrates concepts from psychology, neuroscience, ecology, and bio-

125 geography [2] is thought to drive direct changes in prey distribution and, consequently, indirect

126 changes in lower trophic level resources. Thus, the landscape of fear acts as a buffer to lower

127 trophic levels from over-consumption by other consumers (usually herbivores), and it has been

128 linked to the occurrence of trophic cascades [25]. A well-known example of this concept is the

129 reintroduction of wolves in Yellowstone National Park, whereby the re-insertion of the fear of

130 mortality by wolves has been correlated with changes in elk reproductive fitness, decreases in elk

131 populations, and dramatic changes in the structure of the natural landscapes [12,26-29].

Predator ecologists have suggested that failing to consider the landscape of fear will underestimate the effect that large carnivores play. While this concept is well-established in the

134 ecological community, the costs of the risk of predation are rarely quantified beyond food-risk 135 mesocosm-based approaches [14] or correlations, and they tend to be an inferred construct of the 136 effects of predation risk on prey.

138 beyond the risk of being predated to any space-linked process that may lead to death in a 139 probabilistic sense. Examples include environmentally challenging terrains, such as cliffs for ungulates, or downdraughts for birds, whose value may also change over time, and there is no $a$ 
141 priori reason why such phenomena should not be treated within the same framework.

142 Nevertheless, we concentrate our discourse on 'fear' being used to describe the fear of being

143 killed because, while we know that the risk of being killed in a general sense can affect animal

144 movements (particularly large vertebrates), our understanding of how it actually changes their

145 distribution in space and time is lacking [30,31].

146 Given the above, the landscape of fear remains rather vaguely described, particularly as it

147 related to how species navigate their landscapes. Integrating separate, yet complementary

148 concepts surrounding animal decision-making should provide a more holistic understanding of

149 how energy and fear drive the distribution of free-ranging animals. Here, we present a series of

150 real-world, well-studied examples that empirically demonstrate the integration of both energy

151 landscapes and the landscape of fear (i.e., the risk of being killed). We then provide a

152 hypothetical example for quantifying them together to enhance our understanding of animal

153 distribution.

\section{Intersections between energy and risk in the wild}

155 While it is common to refer to 'the landscape of fear' to describe the constraints that

156 predation risk can create, it is often debatable whether fear or stress are involved, although it is

157 often clear that energetic effects are central to the costs of avoiding predation. Energetic or

158 nutritional costs driven by trade-offs between vigilance and foraging are well described (and

159 often important), but avoiding predation, for example, can influence energetics in less obvious

160 ways.

To illustrate intersections between risk and energetics with consequences for the

162 distribution and abundance of a species, consider the African wild dog (Lycaon pictus). Wild 
163 dogs commonly prey on species like wildebeest (Connochaetes taurinus), warthog (Phacocoerus

164 africanus), gazelles (Gazella spp.) and impala (Aepyceros melampus) that are important prey for

165 much larger carnivores including lions (Panthera leo) and spotted hyenas (Crocuta crocuta)

166 (Crocuta crocuta) [32-34], and this dietary overlap creates the potential for interspecific

167 competition [35-37]. The energetic costs of catching and killing ungulate prey are substantial

168 [38], which creates an additional selection pressure favoring kleptoparasitism, even if live prey

169 are not in limited supply. Consequently, hyenas sometimes follow wild dogs while they are

170 hunting (even before they make a kill) and where hyenas are abundant and visibility is good,

171 hyenas displace wild dogs at up to $86 \%$ of their kills [35,39], although the rate of

172 kleptoparasitism by hyenas varies 43-fold among published studies [40]). These food losses by

173 wild dogs must be offset by increasing the energy invested in finding, catching and killing prey,

174 which is substantial even without losses to scavengers $[38,41]$

175 Moreover, the behavioral and morphological adaptations of large carnivores make the

176 consequences of interference competition potentially severe, and conflict with lions is a common

177 cause of death for wild dogs $[36,42,43]$. As a consequence, wild dogs avoid lions at all spatio-

178 temporal scales (Fig. 1). At the finest scale, the most common response of wild dogs to nearby

179 lion roars is to stop, reverse direction and rapidly move several kilometers away. At the scale of

180 entire ecosystems, wild dog densities are low where lion (and hyena) densities are high [35,42].

181 At intermediate scales, wild dogs consistently avoid areas that are heavily used by lions (Fig.1)

$182[35,36,44]$ and in the Selous Game Reserve, this avoidance of lions has caused wild dogs to hunt

183 most often in deciduous woodland, where their rates of encounter with prey (3.8 prey

184 animals/km moved) are substantially lower than in habitats preferred by lions (9.9 prey

185 animals/km moved) (Fig. 1). 
But these data only show that lions and spotted hyenas limit wild dogs in large part by energetic mechanisms such as food loss, reduced rates of prey encounter, forced changes in travel routes and rapid avoidance movements. We speculate that movement-related energetic consequences of risk avoidance are highly likely to exist beyond this, not least because the environment is different, affecting power costs for movement in a more subtle manner. For example, prey pursuit of prey by wild dogs in deciduous woodland will necessarily involve a more tortuous path than on the open grasslands, with correspondingly increased power use due to the substantial energetic costs of turning [45]. Such effects, summed over several hunts per day 194 and 365 days per year, could have appreciable effects on fitness.

Field studies using GPS collars are beginning to quantify these more subtle interactions 196 between risk and energy (Fig. 2). For example, in a landscape with a mosaic of grassland and 197 forest, the presence of wolves caused elk to shift from preferred foraging habitat in meadows to 198 the protective cover of forests, but in a manner that produced relatively little change in elevation, 199 slope or speed of travel [46]. This reveals that even strong habitat shifts in response to risk can 200 be sensitive to the costs of movement. Even more subtly, foraging locations used by elk were 201 strongly related to snow depth and density when wolves were absent, but not when wolves were 202 present [47]. Because the costs of locomotion and digging for food depend on snow depth and 203 condition, this represents an important interaction between the landscapes of energy and risk 204 (Fig. 2).

To summarize, the costs of antipredator responses are often strongly tied to energetics. A 206 better understanding of this intersection will require simultaneous attention to measurements of 207 risk, foraging success [14] and energy expenditure. 


\section{Box 1. Fear and energy landscapes integrated in mid-air: Herring gulls}

Like all soaring birds, Herring gulls Larus argentatus can accrue great energetic savings

211 by selectively flying in areas with rising air that allows them to glide rather than flap, so it is

212 little surprise that their medium scale movement is partly modulated by the distribution of this

213 lift. However, examining flight trajectories over a fine scale, Shepard et al. [48] have shown that

214 gulls using lift generated by onshore winds hitting seafront hotels do not position themselves in

215 the positions of highest lift, which is immediately adjacent to the buildings. Instead, they fly

216 some 10-20 m away, where the collision risk is reduced but where they can still gain from the,

217 albeit reduced, orographic life. Data presented in the work by Shepard et al. [48] allow some

218 simple calculations to see the extent to which the risk is traded for energy so that energy losses

219 can be equated directly with distances to life-threatening features within the environment (see

220 Box 1 caption for further text).

\section{Integrating concepts}

We propose that we can use least cost pathways within the energy landscape as a

223 mechanism with which to quantify landscape effects because non-concordance of trajectories

224 with a minimal cost solution would indicate prioritization to other aspects, such as reducing the

225 risk of predation. Specifically, the extent of deviations from the minimum path should help our

226 understanding of movement driver hierarchies with the difference in cost between the least cost

227 pathway and that chosen being attributable to the landscape of fear, or indeed any other

228 constraint (Fig. 3). 


\section{Conclusion}

Animal ecology has become increasingly mechanistic in recent years, with researchers

233 applying different paradigms to understand how animals are distributed in space and time.

234 Energy is often termed the currency of life, and animals are expected to use habitats and display

235 movement paths that optimize energy acquisition with direct links to fitness. Yet, if an animal

236 encounters a predator, it may be killed, rendering future (and possibly lifetime depending on the

237 individual circumstances such as life-stage or age or reproductive strategy) fitness zero. Clearly,

238 the concepts of energy acquisition and use related to energy landscapes and potential interaction

239 with predators in the landscape of fear are interacting paradigms that complement each other and

240 collectively provide a more coherent understanding of mechanistic basis of spatial ecology and

241 decision-making within wild animals. The simple models presented here reveal how movement

242 pathways may be modulated by both energy and fear. Although there have been great strides

243 made in conceptualizing animal movement ecology [49], significant research gaps still exist [50].

244 We believe that the integration of the concepts of energy landscapes and landscapes of fear will

245 be a new frontier for understanding animal movement ecology which should help identify

246 important mechanisms modulating the spatial ecology of wild animals.

\section{Acknowledgements}

248 We thank our many colleagues for their thoughtful insights that greatly benefitted this article.

249 Cooke is supported by the Natural Sciences and Engineering Research Council of Canada, Ocean

250 Tracking Network Canada, and the Canada Research Chairs Program. 
255 Figure 1. Energy and fear affect wild dog movement in space and time. Wild dogs are often

256 killed by lions, and as a consequence, (A) wild dogs avoid areas that are heavily used by lions, in

257 many ecosystems. Data shown are utilization distributions from GPS collars in Liuwa Plains

258 National Park, Zambia. Data from 3,271 kilometers of directly observed hunting in the Selous

259 Game Reserve show that (B) wild dogs hunt 52\% more often in areas that are little-used by lions,

260 (C) even though their rate of encounter with prey is decreased by $20 \%$ in such areas.

261

262 Figure 2. Elk responses to wolf predation risk. Responses to predation risk are likely to

263 intersect with energy landscapes in many ways, some overt and some subtle. In response to the

264 presence of wolves, elk become more vigilant and move out of open grasslands where they

265 prefer to forage, but are conspicuous. These strong responses are associated with much smaller

266 changes in elevation, slope, snow depth and speed of travel, suggesting that antipredator

267 responses are shaped by the energetic costs of locomotion and 'cratering' in snow for access to

268 grasses.

269 Figure 3. Quantitative integration of paradigms. Schematic diagram of two possible paths taken

270 by an animal 'intending' to move from A to B [the 'intent' is important here since the movement

271 destination and driver means that the end point is defined - A good example of such a scenario

272 would be a central place forager returning to the central place $\{$ nest/burrow etc. $\}$ at the end of a

273 foraging bout]. The squares denote the cost of transport (COT) for movement and are composed,

274 within the landscape, of low cost squares (' $\mathrm{L}$ ' joules per metre - in grey) and high cost squares

275 ('H' Joules per metre - in red). Normally, if the COT were uniform across the landscape, the least

276 cost pathway between A and B would be a straight line (blue line in the Fig) and the total cost of

277 travel would be LD (where $\mathrm{D}=$ distance). In the case above, there is an area of higher COT so the 
278 animal should follow the dashed black trajectory if $\mathrm{LD}_{1}<\left(\mathrm{LD}_{2}+\mathrm{HD}_{3}\right)$ (where $\mathrm{D}_{1}$ is the distance

279 covered by the black dashed line, $\mathrm{D}_{2}$ is the distance covered by the blue line within the grey square

280 zone and $\mathrm{D}_{3}$ is the distance covered by the blue line within the red square zone). If we insert a

281 predator area which the animal intends to avoid (the red ovoid), the extent of the deviation from

282 the minimum cost path, and specifically its cost, should give us some metric of the perceived

283 danger. This is given by the extra energy that the animal is prepared to spend to reach its goal

284 above the minimum cost. This energy can be summarized as; Energy $=\left(\mathrm{LD}_{4}+\mathrm{HD}_{5}\right)-\mathrm{LD}_{1}$ or

285 Energy $=\left(\mathrm{LD}_{4}+\mathrm{HD}_{5}\right)-\left(\mathrm{LD}_{2}=\mathrm{HD}_{3}\right)$, whichever is the lesser.

286

287 Box 1. Seabirds balance energy and risk in the wild. (A) Schematic diagram of a cross-section

288 of an urban seafront zone (adapted from Shepard et al. in press) showing how a prevailing

289 onshore wind hits the buildings and is forced up creating lift that varies as a function of distance

290 from the constructions. Herring gulls fly within this lift (e.g. black circle in diagram). Actual data

291 on lift and locality can be derived from computational fluid dynamic models - for example for

292 wind speeds of $5.5 \mathrm{~m} / \mathrm{s}$ (presented in Shepard et al. [48]. Assuming that gliding gulls have a

293 power use of about $10 \mathrm{~W}$ [51] and have a glide polar (flight speed versus drop rate) as presented

294 in Shepard et al. (in press), the flight speed of gulls gliding at different distances from the

295 buildings can be calculated knowing that the birds maintain constant height (drop rate = lift rate).

296 (B) In turn, the gliding metabolic rate can be divided by the speed to derive the costs of transport

297 as a function of distance from the buildings). Herring gulls (circles in B) do not fly in the zone of

298 highest lift, which would give them the lowest travel costs, but prefer to occupy a more zone

299 distant where the probability of collision with the buildings is reduced. 


\section{Literature Cited}

3021 Nussbaum MC (1978) Aristotle's De motu animalium: text with translation, commentary and interpretative essay, Princeton University Press.

3042 Laundré, J.W. et al. (2001) Wolves, elk, and bison: reestablishing the "landscape of fear" in Yellowstone National Park, U.S.A. Can. J. Zool. 79, 1401-1409

3063 Stephens, D. et al. (2007) Foraging: behavior and ecology., University of Chicago Press.

3074 Shaw, A. and Kouzin, I. (2013) Migration or residency? The evolution of movement

308 behavior and information usage in seasonal environments. Am. Nat. 181, 114-124

3095 Davies, N. et al. (2012) An Introduction to Behavioural Ecology, John Wiley and Sons.

3106 Charnov, E. (1976) Optimal foraging, the marginal value theorem. Theor. Popul. Biol. 9, $311 \quad 129-136$

3127 Louzao, M. et al. (2014) Coupling instantaneous energy-budget models and behavioural mode analysis to estimate optimal foraging strategy: an example with wandering albatrosses. Mov. Ecol. 2, 8

Weibel, E.R. et al. (2004) Allometric scaling of maximal metabolic rate in mammals: Muscle aerobic capacity as determinant factor. Respir. Physiol. Neurobiol. 140, 115-132

Schmitz, O.J. et al. (2004) Trophic cascades: The primacy of trait-mediated indirect interactions. Ecol. Lett. 7, 153-163

Wilson, R.P. et al. (2012) Construction of energy landscapes can clarify the movement

322 and distribution of foraging animals. Proc. R. Soc. B Biol. Sci. 279, 975-80

Shepard, E.L.C. et al. (2013) Energy landscapes shape animal movement ecology. Am. Nat. 182, 298-312

Creel, S. et al. (2009) Glucocorticoid stress hormones and the effect of predation risk on elk reproduction. Proc. Natl. Acad. Sci. U. S. A. 106, 12388-12393

Christianson, D. and Creel, S. (2010) A nutritionally mediated risk effect of wolves on elk. Ecology 91, 1184-1191

14 Brown, J.S. and Kotler, B.P. (2004) Hazardous duty pay and the foraging cost of predation. Ecol. Lett. 7, 999-1014

15 Bohrer, G. et al. (2012) Estimating updraft velocity components over large spatial scales: Contrasting migration strategies of golden eagles and turkey vultures. Ecol. Lett. 15, 96103

Leichti, F. et al. (2000) Predicting migratory flight altitudes by physiological migration models. Auk 117, 205-214

1 Scantlebury, D.M. et al. (2014) Flexible energetics of cheetah hunting strategies provide resistance against kleptoparasitism. Science 346, 79-82

1 Dickinson, M.H. et al. (2000) How animals move: an integrative view. Science 288, 100- 
Lejeune, T.M. et al. (1998) Mechanics and energetics of human locomotion on sand. $J$. Exp. Biol. 201, 2071-2080

20 White, W.T. and Yousef, M. (1978) Energy expenditure in reindeer walking on roads and on tundra. Can. J. Zool. 56, 215-223

21 Minetti, A.E. et al. (2002) Energy cost of walking and running at extreme uphill and downhill slopes. J. Appl. Physiol. 93, 1039-1046

22 Lima, S.L. and Dill, L.M. (1990) Behavioral decisions made under the risk of predation: a review and prospectus. Can. J. Zool. 68, 619-640

Estes, J.A. et al. (2011) Trophic downgrading of planet Earth. Science 333, 301-306

Laundre, J.W. et al. (2010) The Landscape of Fear: Ecological Implications of Being Afraid !2009-09-09 !2009-11-16 !2010-02-02 ! Open Ecol. J. 3, 1-7

Suraci, J.P. et al. (2016) Fear of large carnivores causes a trophic cascade. Nat. Commun. 7,10698

Ripple, W.J. and Beschta, R.L. (2003) Wolf reintroduction, predation risk, and cottonwood recovery in Yellowstone National Park. For. Ecol. Manage. 184, 299-313

Fortin, D. et al. (2005) Wolves influence elk movements: Behavior shapes a trophic cascade in Yellowstone National Park. Ecology 86, 1320-1330

Creel, S. et al. (2007) Predation Risk Affects Reproductive Physiology and Demography of Elk. Science 315, 960

Winnie, J.A. (2012) Predation risk, elk, and aspen: tests of a behaviorally mediated trophic cascade in the Greater Yellowstone Ecosystem. Ecology 93, 2600-2614

Hebblewhite, M. and Merrill, E. (2009) Trade-offs between predation risk and forage differ between $\mathrm{m}$ igrant strategies in a migratory ungulate. Ecology 90, 3445-3454

33 Estes, R. and Goddard, J. (1967) Prey selection and hunting behavior of the African wild Werner, E.E. et al. (2015) An Experimental Test of the Effects of Predation Risk on Habitat Use in Fish. 64, 1540-1548

Creel, S. and Creel, N. (1995) Communal hunting and pack size in African wild dogs,

34 Hayward O’Brien, J., Hofmeyr, M., Kerley, G.I.H., M.W. et al. (2006) Prey preferences of the African wild dog Lycaon pictus: ecological requirements for their conservation. $J$. Mammal. 87, 1122-1131

Creel, S. and Creel, N. (1996) Limitation of African Wild Dogs by Competition Limitation with Larger Carnivores. Conserv. Biol. 10, 526-538

36 Mills, M. and Gorman, M. (1997) Factors affecting the density and distribution of wild 
401

402

403

404

dogs in the Kruger National Park. Conserv. Biol. 11, 1397-1406

37 Owen-Smith, N. and Mills, M.G.L. (2008) Predator-prey size relationships in an African large-mammal food web. J. Anim. Ecol. 77, 173-183

38 Gorman, M.L. et al. (1998) High hunting costs make African wild dogs vulnerable to kleptoparasitism by hyaenas. Nature 852, 1992-1994

39 Fanshawe, J. and Fitzgibbon, C. (1993) Factors influencing the hunting success of an African wild dog pack. Anim. Behav. 45, 479-490

40 Creel, S. et al. (2001) Interspecific competition \& population biology of extinction-prone carnivores. In Carnivore Conservation (Gittleman, J. et al., eds), pp. 35-61, Cambridge

41 Creel, S. (1997) Cooperative hunting and group size: assumptions and currencies. Anim. Behav. 54, 1319-24

42 Creel, S. and NM, C. (2002) The African Wild Dog: Behavior, Ecology and Conservation, Princeton University Press.

43 Woodroffe, R. et al. (2007) Rates and causes of mortality in Endangered African wild dogs Lycaon pictus: lessons for management and monitoring. Oryx 41, 215-233

44 Swanson, A. et al. (2014) Cheetahs and wild dogs show contrasting patterns of suppression by lions. J. Anim. Ecol. 83, 1418-1427

45 Wilson, R. et al. (2013) Turn costs change the value of animal search paths. Ecol. Lett. 16, $1145-1150$

46 Creel, S. et al. (2005) Elk alter habitat selection as an antipredator response to wolves. Ecology 86, 3387-3397

47 Winnie, J.A. et al. (2006) Elk decision-making rules are simplified in the presence of wolves. Behav. Ecol. Sociobiol. 61, 277-298

48 Shepard, Emily L C, Williamson, C. and Windsor, S.P. Fine-scale flight strategies of gulls in urban airflows indicate risk and reward in city living. Philos. Trans. R. Soc. Lond. B. Biol. Sci.

49 Holyoak, M. et al. (2008) Movement Research. Proc. Natl. Acad. Sci. 105, 19052-19059

Holyoak, M. et al. (2008) Trends and missing parts in the study of movement ecology. Proc. Natl. Acad. Sci. U. S. A. 105, 19060-19065

51 Baudinette, R. and Schmidt-Nielsen, K. (1974) Energy cost of gliding flight in herring gulls. Nature 248, 83-84 